Arne Heise, Toralf Pusch

\title{
Die „Harmonie der Täuschungen“ muss enden, damit Politikberatung glaubwürdiger wird
}

\begin{abstract}
Als die Bundesregierung beschloss, in Deutschland einen Mindestlohn von 8,50 Euro zum 1.1.2015 einzuführen, tat sie dies gegen den fast einmütigen Rat der wirtschaftswissenschaftlichen Fachleute. Die großen wirtschaftswissenschaftlichen Forschungsinstitute, die Mehrheitsmeinung des Sachverständigenrats (SVR) und fast alle weiteren Stimmen der universitären Ökonomie warnten vor einem großen sozialen Experiment, das voraussichtlich mit Beschäftigungseinbußen von bis zu 1 Mio. Arbeitsplätzen und entsprechendem Anstieg der Arbeitslosigkeit - insbesondere im Bereich der geringqualifizierten Beschäftigung - bezahlt werden müsste (DIW et al., 2008; Knabe, Schöb und Thum, 2014; SVR, 2014, 284 ff.).
\end{abstract}

\section{Das Waterloo der Arbeitsmarktökonomik}

Die weitgehende Einmütigkeit der Ökonom:innen - lediglich einige Non-Mainstreamer und das von den Gewerkschaften nominierte SVR-Mitglied kamen zu anderen, weniger wohlfahrtsgefährdenden Einschätzungen (Herr, Kazandziska und Mahnkopf-Praprotnik, 2009; SVR, 2014, $44 \mathrm{f}$.) - war gleichermaßen absehbar wie unverständlich. Sie war absehbar, weil ein positiver Preis-Mengen-Zusammenhang (oder hier eben Lohn-Beschäftigungs-Zusammenhang) geradezu konstituierend für die tradierte Markttheorie ist und deshalb ein Mindestlohn, der über dem markträumenden Gleichgewichtslohn liegt (und ein Mindestlohn darunter wäre unsinnig), letztendlich immer zu Beschäftigungseinbußen und Arbeitslosigkeit führen

(C) Der/die Autor:in 2021. Open Access: Dieser Artikel wird unter der Creative Commons Namensnennung 4.0 International Lizenz veröffentlicht (creativecommons.org/licenses/by/4.0/deed.de).

Open Access wird durch die ZBW - Leibniz-Informationszentrum Wirtschaft gefördert.

Prof. Dr. Arne Heise ist Professor für VWL, insbesondere Finanzpolitik und Public Governance am FB Sozialökonomie der Universität Hamburg.

Dr. Toralf Pusch ist Referatsleiter am wirtschaftsund Sozialwissenschaftlichen Institut (WSI) der Hans Böckler Stiftung in Düsseldorf. muss. Je nach Modellvariante (wettbewerblicher Arbeitsmarkt, eingeschränkte Konkurrenz, mit oder ohne Transaktionskosten, etc.) kann dieser Effekt unterschiedlich groß sein, aber er muss signifikant ausfallen, sonst ist die Markttheorie im Kern erschüttert.

Andererseits erstaunt die Einmütigkeit doch, weil zumindest die Arbeitsmarktfachleute unter den Ökonom:innen die internationale Forschung zur Empirie des Mindestlohns hätten kennen müssen: Da Mindestlöhne weltweit in vielen Ländern teilweise seit vielen Jahren existieren und massiv beforscht wurden, existieren nicht nur zahlreiche Studien zum Einfluss des Mindestlohns auf die Beschäftigung, sondern auch Meta-Studien, welche die einzelnen Studien auswerten und gemeinsame Erkenntnisse zu gewinnen versuchen. Das Ergebnis hat der renommierte Arbeitsmarktökonom Alan Manning (2021) erst jüngst mit dem Ausdruck elusive - schwer fassbar, flüchtig - belegt: Der von fast allen erwartete negative Beschäftigungseffekt lässt sich einfach in der weltweiten Realität der sehr unterschiedlichen Mindestlohnsysteme nirgends nachweisen. Gewiss kann man davon sprechen, dass dieses empirische Ergebnis eine schwere Anomalie darstellt und die Erklärungskraft und Prognosefähigkeit der verschiedenen Varianten der tradierten Arbeitsmarkttheorie unterminiert, doch hat dies die große Mehrheit der Ökonom:innen weder in ihrem Glauben an die Theorie erschüttert, noch dazu gebracht, in ihrer Politikberatung vorsichtiger oder besser, redlicher zu werden. Vielmehr wurde die überwältigende empirische Evidenz damit abgetan, dies habe keine Aussagekraft für Deutschland, da der deutsche Arbeitsmarkt anders sei und die Höhe des anvisierten Mindestlohns höher sei, als dies bislang beobachtet wurde.

Wir wissen, dass die Politik sich letztlich nicht davon hat abschrecken lassen, den flächendeckenden Mindestlohn in Deutschland einzuführen. Allerdings hat sie eine Begleitforschung installiert, um im Bedarfsfall Korrekturen vorzunehmen, und die dynamische Gestaltung der Mindestlohnhöhe wurde „entpolitisiert", indem sie einer Mindestlohnkommission bestehend aus einem "neutralen" Mitglied, jeweils drei Mitgliedern der Arbeitgeber- und der Arbeitnehmerseite und zwei stimmlosen Forschenden übergeben wurde. Die Begleitforschung ist zum Waterloo der deutschen Arbeitsmarktforschung geworden, denn sie hat deren Befürchtungen massiver Arbeitsplatzverluste widerlegt und damit die bereits bekannten Ergebnisse 
der internationalen empirischen Mindestlohnforschung nur bestätigt: Auch in Deutschland ist der Beschäftigungseffekt des Mindestlohns „flüchtig“ (unter anderem Bruttel, Baumann und Dütsch, 2019)!

\section{Wie reagieren Wissenschaft und Politikberatung?}

Wie nun reagiert die Wirtschaftswissenschaft in Forschung und Politikberatung auf dieses Fiasko? Wissenschaftsphilosophisch geschulte Fachfremde könnten vermuten, dass die empirische Anomalie als Beginn eines Umdenkens aufgefasst und nach paradigmatischen Alternativen - die ja vorhanden sind - gesucht wird (Paradigmenwechsel). Doch weit gefehlt - die tradierte Ökonomik (übrigens nicht nur in Deutschland) geht andere Wege: Einerseits werden Modellergänzungen und -erweiterungen entwickelt, welche die Empirie mangelnder Beschäftigungseffekte von Mindestlöhnen mit dem allokativen Ansatz des tradierten Arbeitsmarktmodelles ex post versöhnen könnten (unter anderem Braun et al., 2020, die diesen Anspruch von den Ergebnissen ihrer Prognose her allerdings nicht einlösen können). Andererseits wird nach alternativen Anpassungswegen gesucht, die Unternehmen statt einer Beschäftigungsreduktion gewählt haben könnten (unter anderem Bossler et al., 2018). Und schließlich wird nachzuweisen versucht, dass die Prognose massiver Beschäftigungsverluste doch eingetreten sei, aber nicht recht erkannt werde (Knabe, Schöb und Thum, 2020). Es macht sich also bemerkbar, was der Wissenschaftsphilosoph Ludwik Fleck (1980) einmal „Denkstilzwang“ nannte und eine unglaubliche Resilienz der tradierten Ökonomik beschreibt.

Allerdings gelingt es keinem dieser Verteidigungsversuche allein, eine Ex-Post-Prognose zu liefern, die der Realität entspricht (Heise, 2021). Natürlich könnte der Stein der Weisen darin liegen, die verschiedenen Herangehensweisen zu kombinieren - also z. B. ein Monopson-Modell mit Transaktionskosten, alternativen Anpassungswegen und versteckten Effekten -, um so gerade und gegebenenfalls etwas zufällig einen neutralen Beschäftigungseffekt des Mindestlohns zu konstruieren. Aber wieso sollte diese Kombination in allen anderen Ländern mit all ihren unterschiedlichen Mindestlohnsystemen und Mindestlohnhöhen, in denen die empirische Mindestlohnforschung ja auch keinen Beschäftigungseffekt hat aufdecken können, ebenfalls gerade so ausfallen, dass keine Jobverluste und keine Arbeitslosigkeit entstehen? Wer auf all diese Zufälligkeiten baut, könnte leicht dem erliegen, was Ludwik Fleck eine „Harmonie der Täuschungen“ genannt hat.

Und welche Konsequenzen hat die Politikberatung daraus gezogen, sich beim letzten Mal eine ziemlich blutige Nase geholt zu haben? Auffällig ist, dass sich die großen Forschungsinstitute mit konkreten Simulationen einer Erhöhung des Mindestlohns auf die von der potenziellen Ampelkoalition befürworteten 12 Euro pro Stunde zurückhalten und allenfalls tentative Aussagen machen. Immerhin befürwortet DIW-Präsident Marcel Fratzscher (2019) die Erhöhung explizit mit dem Verweis auf nicht zu erwartende Beschäftigungs-, sehr wohl aber zu erwartende Einkommenseffekte.

Ifo-Präsident Clemens Fuest (2018) hingegen - und ähnlich lassen sich Forschende des Instituts für Arbeitsmarkt- und Berufsforschung (IAB) der Bundesagentur für Arbeit zitieren (Bossler, Oberfichtner und Schnabel, 2018) - rät wiederum ungerührt durch die vorliegenden Forschungsergebnisse von einer Steigerung auf 12 Euro $a b$, weil er trotz alledem dramatische Beschäftigungseffekte befürchte. Eine angemessene Aufarbeitung wissenschaftlicher Ergebnisse sieht sicher anders aus.

\section{Zwölf Euro Mindestlohn}

Wenngleich die Befürwortenden einer Mindestlohnsteigerung sicher gegenwärtig die besseren Argumente haben als die Gegenseite, sollte man doch nicht dem Trugschluss verfallen, Erhöhungen des Mindestlohns könnten niemals zu Beschäftigungsverlusten führen. Selbst wenn Kreislaufeffekte grundsätzlich Allokationseffekte dominieren mögen, wird wohl niemand abstreiten, dass eine Mindestlohnerhöhung spätestens dann zu Beschäftigungsverlusten führt, wenn die gesamtwirtschaftliche Nachfrage leidet - sei es durch restriktive geldpolitische Reaktionen, preisliche Wettbewerbsprobleme in einem gemeinsamen Währungsraum oder auch durch rückläufige Unternehmensinvestitionen.

Ob dies der Fall ist bei einer Erhöhung des Mindestlohns von gegenwärtig 9,60 Euro auf 12 Euro pro Stunde $(=+25 \%)$, die insgesamt ca. 9 Mio. Angestellten betreffen und eine durchschnittliche Stundenlohnerhöhung um $3 \%$ bedeuten würde (vgl. Tabelle 1), ließe sich beispielsweise dann sagen, wenn Simulationen in Erweiterung jener heterodoxen Modelle bereitgestellt werden könnten, die bereits bei einfachster Modellierung Ergebnisse zeigten (Heise und Pusch, 2020), die der Realität sehr nahe kamen - leider gibt es solche elaborierten Simulationsmodelle mangels wissenschaftlicher Kapazitäten und finanzieller Ressourcen (noch) nicht.

Dennoch erscheint es nicht sehr wahrscheinlich, dass ein Mindestlohn von 12 Euro tatsächlich bereits so hoch wäre, dass gravierende Beschäftigungsverluste befürchtet werden müssten; nachfrageseitig ist bei Mindestlohn-Beschäftigten im Schnitt mit einer höheren Konsumneigung und -ausgaben zu rechnen, allokationstheoretisch fallen 
Tabelle 1

Hochgerechnete Verbreitung von Stundenlöhnen unter 12 Euro im Jahr 2021

\begin{tabular}{|c|c|c|c|c|}
\hline & $\begin{array}{l}\text { Beschäftigte } \\
\text { bzw. Jobs }\end{array}$ & $\begin{array}{r}\text { davon ur } \\
12 \text { Eur }\end{array}$ & $\begin{array}{l}\text { ter } \\
\text { o }\end{array}$ & $\begin{array}{c}\text { Steigerung } \\
\text { Stunden- } \\
\text { lohn (in \%) } \\
\text { bei } 12 \text { Euro } \\
\text { Mindest- } \\
\text { lohn }\end{array}$ \\
\hline $\begin{array}{l}\text { Alle Beschäftigten } \\
\text { (Hauptjob) }\end{array}$ & 33.565 .000 & 7.326 .000 & 21,8 & 2,8 \\
\hline Männer & 16.926 .000 & 2.739 .000 & 16,2 & 2,0 \\
\hline Frauen & 16.102 .000 & 4.423 .000 & 27,5 & 3,8 \\
\hline Vollzeit & 22.634 .000 & 3.045 .000 & 13,5 & 1,4 \\
\hline Teilzeit & 10.930 .000 & 4.280 .000 & 39,2 & 6,8 \\
\hline Minijobs & 3.192 .000 & 2.549 .000 & 79,9 & 31,4 \\
\hline Mit Tarif & 15.978 .000 & 1.512 .000 & 9,5 & 1,0 \\
\hline ohne Tarif & 14.399 .838 & 4.304 .000 & 29,9 & 4,1 \\
\hline Nebenjobs: alle & 2.243 .000 & 1.327 .000 & 59,2 & 12,0 \\
\hline
\end{tabular}

Quellen: Pusch (2021) auf Basis von SOEP v36 und Daten des Statistischen Bundesamts.

die Nachfragerückgänge von Produkten typischer Niedriglohnsektoren aufgrund einer verhältnismäßig geringen Preiselastizität der Nachfrage im Schnitt eher gering aus (Heise und Pusch, 2020).

Eine Beschäftigungsreaktion in Folge eines Mindestlohns von 12 Euro wäre wie schon bei der Einführung des gesetzlichen Mindestlohns am ehesten bei den geringfügig Beschäftigten zu erwarten. Dies dürfte aber in vielen Fällen damit zusammenhängen, dass geringfügige Beschäftigung in sozialversicherungspflichtige Beschäftigung umgewandelt wird. Außerdem bezieht etwa die Hälfte der geringfügig Beschäftigten soziale Transfers mit Zuverdienstgrenzen, die sich häufig an der Minijob-Verdienstgrenze orientieren (Pusch et al., 2021, 119).

Allein aus diesem Grund ist eine Umwandlung von Minijobs in höher entlohnte sozialversicherungspflichtige Beschäftigung für viele von innen nicht attraktiv. Als Alternative bleiben dann Arbeitszeitverkürzungen oder, falls das für die Arbeitgeber:innen organisatorisch nicht umsetzbar ist, auch die Aufgabe der Beschäftigung. Auf jeden Fall treffen Beschäftigungsrückgänge bei den geringfügig Beschäftigten auch angesichts der geringen Verdienste nicht den Kern des Arbeitsmarktes.

Aus dem zurückliegenden Fiasko der Politikberatung sollte gelernt werden, nicht nur die „flüchtigen“ Beschäftigungseffekte des Mindestlohns zum Gradmesser einer
Empfehlung zu machen, sondern auch die Auswirkungen auf die Einkommensverteilung (Pusch et al., 2021) und Armutsentwicklung ${ }^{1}$ oder die Arbeitszufriedenheit und -produktivität zu berücksichtigen (Pusch und Rehm, 2017).

1 Die Mindestlohnhöhe von 12 Euro ist ja nicht zufällig gewählt, sondern entspricht etwa jenem Stundenlohn, den ein/e Vollzeitarbeitnehmer:in mit normaler Erwerbsbiografie (45 Jahre Vollzeitarbeit) etwa verdienen müsste, um im Alter nicht unter die Grundsicherung zu fallen.

\section{Literatur}

Bossler, M., N. Gürtzgen, B. Lochner, U. Betzl, L. Feist und J. Wegmann (2018), Auswirkungen des gesetzlichen Mindestlohns auf Betriebe und Unternehmen, Studie im Auftrag der Mindestlohnkommission, Institut für Arbeitsmarkt- und Berufsforschung.

Bossler, M., M. Oberfichtner und C. Schnabel (2018), Employment Adjustments Following Rises and Reductions in Minimum Wages: New Insights from a Survey Experiment, IZA Discussion Paper, Nr. 11747.

Braun, H., R. Döhrn, M. Krause, M. Micheli und T. Schmidt (2020), Macroeconomic Long-Run Effects of the German Minimum Wage when Labor Markets are Frictional, Jahrbücher für Nationalökonomie und Statistik, 240(2-3), 351-386.

Bruttel, O., A. Baumann und M. Dütsch (2019), Beschäftigungseffekte des gesetzlichen Mindestlohns: Prognosen und empirische Befunde; Perspektiven der Wirtschaftspolitik, 20(3), 237-253.

DIW et al. (2008), Gemeinsamer Aufruf der Präsidenten und Direktoren der Wirtschaftsforschungsinstitute vom 12. März 2008, ifo Schnelldienst, 61(6), 3-4.

Fuest, C. (2018), Der gefährliche Überbietungswettbewerb beim Mindestlohn, Wirtschaftswoche, 23. November.

Fleck, L. (1980), Entstehung und Entwicklung einer wissenschaftlichen Tatsache. Einführung in die Lehre vom Denkstil und Denkkollektiv, Frankfurt (Erstausgabe 1935).

Fratzscher, M. (2019), Ist ein Mindestlohn von zwölf Euro sinnvoll?, Zeit Online vom 27. Dezember, https://www.zeit.de/wirtschaft/2019-12/ gehalt-mindestlohn-entgelt-erhoehung-niedriglohnsektor/ komplettansicht?print) (9. November 2021).

Heise, A. (2021), How did they get it so wrong? Mindestlöhne und ihre Bedrohung für die Standardökonomie, ZÖSS Discussion Paper, Nr. 85, Universität Hamburg.

Heise, A. und T. Pusch (2020), Introducing minimum wages in Germany employment effects in a post Keynesian perspective, Journal of Evolutionary Economics, 30(5), 1515-1532.

Herr, H., M. Kazandziska und S. Mahnkopf-Praprotnik (2009), The theoretical debate about minimum wages, Global Labor University working papers, Nr. 6 .

Knabe, A., R. Schöb und M. Thum (2014), Der flächendeckende Mindestlohn, Perspektiven der Wirtschaftspolitik, 15(2), 133-157.

Knabe, A., R. Schöb und M. Thum (2020), Prognosen und empirische Befunde: Wie groß ist die Kluft beim Mindestlohn wirklich?, Perspektiven der Wirtschaftspolitik, 21(1), 25-29.

Manning, A. (2021), The Elusive Employment Effect of the Minimum Wage, Journal of Economic Perspectives, 35(1), 3-26.

Pusch, T. (2021), 12 Euro Mindestlohn. Deutliche Lohnsteigerungen vor allem bei nicht tarifgebundenen Beschäftigten, WSI Policy Brief, Nr. 62.

Pusch, T., V. Tobsch, T. Schmidt und C. Santoro (2021), Effekte des gesetzlichen Mindestlohns auf die Haushaltsnettoeinkommen und den Bezug bedarfsgeprüfter Transferleistungen, WSI-Mitteilungen, 74(2), 116-126.

Pusch, T. und M. Rehm (2017), Positive Effekte des Mindestlohns auf Arbeitsplatzqualität und Arbeitszufriedenheit, Wirtschaftsdienst, 97(6), 409-414, https://www.wirtschaftsdienst.eu/inhalt/jahr/2017/heft/6/ beitrag/positive-effekte-des-mindestlohns-auf-arbeitsplatzqualitaetund-arbeitszufriedenheit.html (22. November 2021).

SVR (2014), Mehr Vertrauen in Marktprozesse, Jahresgutachten 2014/15, Wiesbaden. 